\title{
Breast cancer: Molecular basis and therapeutic strategies (Review)
}

\author{
GIOVANNI LIGRESTI ${ }^{1}$, MASSIMO LIBRA ${ }^{1}$, LOREDANA MILITELLO ${ }^{1}$, SILVIA CLEMENTI $^{1}$, MARCO DONIA ${ }^{1}$, \\ ROSA IMBESI $^{1}$, GRAZIA MALAPONTE ${ }^{1}$, ALESSANDRO CAPPELLANI ${ }^{2}$, \\ JAMES A. MCCUBREY ${ }^{3}$ and FRANCA STIVALA ${ }^{1}$ \\ ${ }^{1}$ Department of Biomedical Sciences, University of Catania; ${ }^{2}$ Department of Surgery, School of Medicine, University of \\ Catania, Catania, Italy; ${ }^{3}$ Department of Microbiology and Immunology, East Carolina University, Greenville, NC, USA
}

Received March 24, 2008; Accepted May 13, 2008

\begin{abstract}
Breast cancer is the most common malignancy among women. It is frequently treated with chemotherapy and hormone therapy. More recently, however, 'targeted therapy' has emerged as an important approach to cancer therapy. Targeted therapy works by interfering with a specific molecular target, though inter-individual variability in drug response often causes treatment failure. Anticancer agents inhibit breast cancer progression by several different mechanisms. The Ras/Raf/MEK/ERK signal transduction pathway regulates cell cycle progression and apoptosis in diverse cell types. Alterations in this pathway are often associated with human cancer, including breast cancer. Understanding breast cancer biology is useful for the identification of appropriate anticancer drugs. This review describes the effect of gene alterations on breast cancer development. In addition, it shows how each anticancer drug used to treat breast cancer may block aberrant cell proliferation. Finally, the mechanisms of resistance to therapy are also discussed.
\end{abstract}

\section{Contents}

1. Introduction

2. Breast cancer biology

3. Chemotherapy

4. Hormone therapy

5. Antibody-based therapy

6. Mechanisms of resistance and the Raf/MEK/ERK pathway

7. Conclusions

\section{Introduction}

Breast cancer is the most common malignancy among women (1). Over 180,000 new cases are diagnosed in the United States

Correspondence to: Dr Massimo Libra, Department of Biomedical Sciences, University of Catania, Via Androne 83, I-95124 Catania, Italy

E-mail: mlibra@unict.it

Key words: breast cancer, hormone therapy, antibody-based therapy, raf each year, and approximately half of all patients diagnosed die from the disease (2). The incidence of breast cancer presents a wide geographic variability; it is almost 10 times more common in the developed countries of the West than in the third world. The rate of incidence in the US in 2005 was 242,570 new cases (32\% of neoplasia diagnosed in women) (3). However, in the last decade, in spite of its increasing incidence, breast cancer mortality has been declining in the majority of developed countries. Undoubtedly, the reasons for this decline are multifactorial: the result of better education, widespread screening programmes and more efficacious adjuvant treatments (4). However, the use of systemic therapies in early disease treatment is commonly regarded as a major contributing factor (5).

An increased knowledge of the regulation of cell growth and the genetic and biochemical changes that lead to malignancy has created many new opportunities for the development of cancer drugs. These new targets include oncogenes, growth factors and their receptors, signal transduction pathways and cell differentiation signals (6).

\section{Breast cancer biology}

Mutations in a number of genes are now known to cause susceptibility to breast cancer. In high-risk families, the most significant of these are the BRCA1 and BRCA2 genes $(7-12,14)$. Hereditary breast cancer accounts for only $\sim 10 \%$ of all breast cancer cases and generally results from the lack of a tumor suppressor gene, as opposed to the gain of an oncogene (13).

BRCA 1 and BRCA 2 are responsible for $80-90 \%$ of genetically determined tumors, codifying nuclear proteins involved in the biochemical mechanisms that control the integrity of the genome. These genes are rarely mutated in sporadic forms, suggesting they play a pathogenetic role in the promotion of carcinogenesis. BRCA genes are also involved in male breast cancer. Men who carry germline mutations in the BRCA2 gene have a higher risk of developing breast carcinoma than men in the general population, and those who carry germline mutations in the BRCA1 gene may also be at a higher risk, though this association is not as well established (15).

Other genes are involved in the genesis of breast cancer as well. These are tumor suppressor genes, p53 and PTEN. The most common route by which the $\mathrm{p} 53$ pathway is inactivated in human cancers is by mutation in the coding region of the 
p53 gene (16). p53 is a transcription factor that regulates the cell cycle and DNA repair. Existing in the cell at a very low level and in a relatively inactive form, p53 protein is increased and activated during periods of cellular stress. The net effect of this increase and activation is the arrest of the cell in cycle, which allows time for the incurred damage to be repaired or for the apoptosis or death of the cell. The failure of these normal protective and adaptive mechanisms, caused by the mutation of the p53 gene and the production of an abnormal protein, permits DNA-damaged cells to continue replicating (17). Germline mutations of p53 result in a cancer predisposition syndrome known as Li-Fraumeni (18), which is associated with brain tumors, sarcomas, leukemia, lymphoma, malignant melanoma and adrenal cortical carcinoma (19).

Phosphatase and tensin homolog (PTEN), a tumor suppressor gene located at human chromosome 10q23, may play an important role in cell proliferation, the cell cycle and the apoptosis of cancer cells (20). Germline mutations of PTEN are associated with Cowden syndrome (CS), a multihamartomatous disorder and rare Mendelian syndrome that includes as part of its phenotype breast cancer (22), an increased risk of thyroid and endometrial cancers, and benign manifestations (23). CS patients with germline PTEN promoter mutations have aberrant PTEN protein expression and an increased frequency of breast cancer (21). PTEN has a function in tumor suppression, a nuclear function that controls chromosomal integrity. Disruption of PTEN leads to extensive centromere breakage and chromosomal translocations (24). Altered apoptotic signal cascades, such as PTEN deletions, may prevent the antitumoral effects of therapy (25).

Relatively common mutations at the level of low-penetrance genes act together with endogenous factors and conditions related to lifestyle in the determinism of the sporadic tumors which comprise most breast carcinomas. A cell with a deficiency in the DNA repair process is assumed to be more exposed to mutagenic insults and to have a higher probability of transforming into a cancer cell. For example, a higher frequency of polymorphisms of the XRCC 1 and XRCC 3 genes was found in breast cancer patients (26). Another example of the relationship between lifestyle and genetic polymorphisms involves alcohol dehydrogenase (ADH) activity. Moderate alcohol consumption has been associated with a 30-50\% increase in breast cancer risk. Individuals differ in their ability to metabolize alcohol through genetic differences in $\mathrm{ADH}$, the enzyme that catalyzes the oxidation of $\sim 80 \%$ of ethanol to acetaldehyde, a known carcinogen. Individuals differ in their $\mathrm{ADH}$ genotype; one locus in particular $(\mathrm{ADH} 3)$ is polymorphic in Caucasian populations. As compared to those who are slow metabolizers, individuals with the ADH3(1-1) genotype have a higher risk of breast cancer due to alcohol intake, even when both consume similar amounts of alcohol (27).

HER2. HER2/neu is a member of the erbB-like oncogene family and is related to, but distinct from, epidermal growth factor receptor (EGFR) (28) (Fig. 1). This oncogene has been shown to be amplified in human breast cancer cell lines, and many studies indicate a close correlation between its expression and prognosis in breast cancer (29). The HER $2 /$ neu proto-oncogene encodes a growth factor receptor that is overexpressed in $20-30 \%$ of metastatic breast cancers. This over-

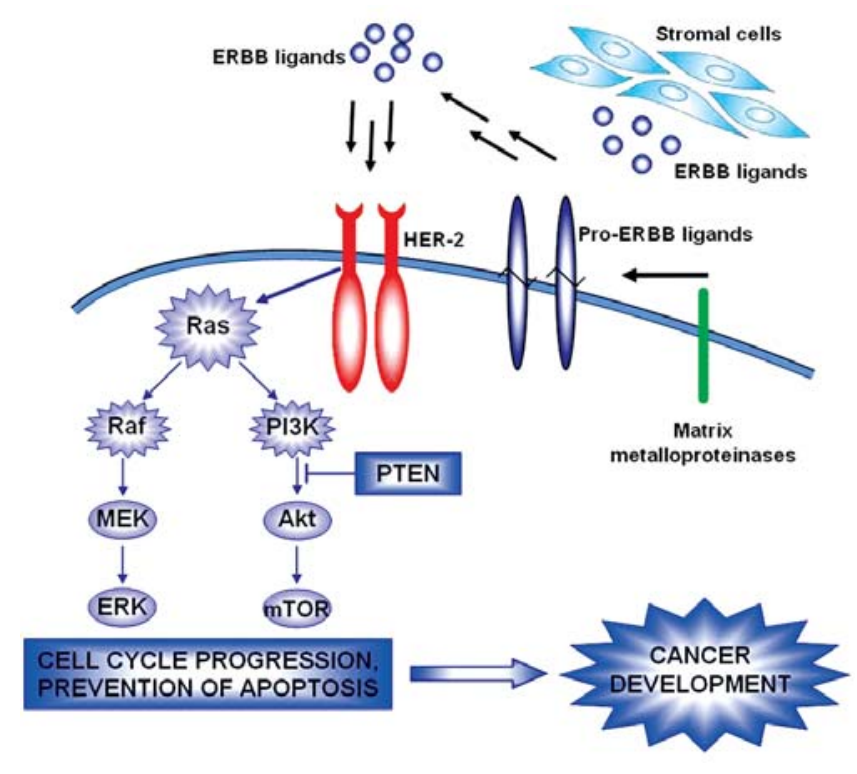

Figure 1. ERBB receptor tyrosine kinases are activated by several mechanisms which comprise mutation, overexpression and the production of epidermal growth factor (EGF) family ligands. ERBB ligands are released from stromal cells and/or produced by the activation of oestrogen receptor, which causes the matrix metalloproteinase-mediated cleavage and release of pro-EGF. Ras, Raf, PI3K and PTEN are the sites of gene alterations which result in the activation of the Raf/MEK/ERK and PI3K/Akt cascades.

expression is associated with decreased survival and decreased relapse-free periods. The anti-HER2 antibody is designed to block this receptor. Epidermal growth factor (EGF), its cognate receptor (EGFR) and related family members have been shown to be important in the normal as well as the malignant growth of many cell types, including glioblastomata, astrocytomas, medulloblastomata, non-small cell lung carcinoma and breast cancer (30).

HER2 gene amplification is a complex event including the coamplification of other potentially oncogenic genes and the facilitation of the generation of additional genomic aberrations (31). It is estimated that amplification of the HER2 gene takes place in roughly $18-20 \%$ of breast cancer patients, resulting in aberrant overexpression of p185HER2 protein. As a result, HER2 represents an attractive therapeutic target (32).

Ras/Raf/MEK/ERK pathway. The Ras/Raf/MEK/ERK cascade couples signals from cell surface receptors to transcription factors, which regulate gene expression. This cascade also regulates the activity of many proteins involved in apoptosis. The pathway is frequently activated in certain tumors by chromosomal translocations such as BRC-ABL, mutations in cytokine receptors such as Flt-3, Kit and Fms, or overexpression of wild-type or mutated receptors, such as EGFR. Abnormal activation of the Raf/MEK/ERK pathway occurs in human cancer due to mutations in the upstream membrane receptors Ras and B-Raf, as well as in genes in other pathways (e.g., PI3K, PTEN, Akt) that serve to regulate Raf activity (33). Mutations at B-Raf are frequently detected in certain malignancies, including melanoma and thyroid cancer (34).

The mitogen-activated protein kinase (MAPK) signaling pathway is a potential target for cancer therapy (35). HER2/ 
neu overexpression can activate PI3K and the Ras/Raf/ MEK/ERK pathway, resulting in the reduction of wild p53 protein expression. This may be the molecular mechanism responsible for the poor prognosis and therapeutic nonresponsiveness of patients with HER2/neu-overexpressed breast cancer (36). The Ras/Raf/MEK/ERK and PI3K/PTEN/ AKT signaling cascades play critical roles in the transmission of signals from growth factor receptors, regulating gene expression and preventing apoptosis. Components of these pathways are mutated or aberrantly expressed in human cancer (e.g., Ras, B-Raf, PI3K, P, Akt) and mutations occur in genes encoding upstream receptors (e.g., EGFR and Flt-3), while chimeric chromosomal translocations (e.g., BCR-ABL) transmit their signals through the cascades. The pathways interact to regulate growth and, in some cases, tumorigenesis (37) (Fig. 1).

PI3K/AkT pathway. PI3K consists of an $85-\mathrm{kDa}$ regulatory subunit containing the SH2 and SH3 domains, and a $110-\mathrm{kDa}$ catalytic subunit (38). The PI3K/Akt signal transduction cascade has been extensively investigated for its roles in oncogenic transformation; uncovering the signaling network spanning the region from the extracellular environment to the nucleus should illuminate biochemical events contributing to malignant transformation. PI3K/Akt signaling plays an important role in regulating the proteins that control cellular proliferation. These targets include cyclins, cyclin-dependent kinases and cyclin-dependent kinase inhibitors (39). PIK3CA mutations are common in invasive ductal carcinomas of the breast (40).

Numerous studies have demonstrated that breast cancer arising in young women is characterized by a higher incidence of negative prognostic factors, higher recurrence rates and poorer overall survival, despite aggressive therapies (41). In fact, breast cancer patients younger than 35 years have a poorer prognosis than older patients (42). The poor prognosis subgroup of young women with breast cancer is characterized by deregulation of the PI3K, Myc and B-catenin pathways. Of these, therapies are available for the PI3K pathway. A strategy which might be applied to this group of patients is therefore PI3K-specific therapy (43).

PI3K/AkT and Ras/Raf/MEK/ERK interactions. The Ras/Raf/ MEK/ERK and Ras/PI3K/PTEN/Akt pathways interact with each other to regulate growth and, in some cases, tumorigenesis. Both pathways also interact with the p53 pathway (33), and contribute to the transcriptional regulation of Bcl-2 family members by regulating CREB phosphorylation. CREB binds the Mcl-1 and Bcl-2 promoter regions (44). Thus, the Raf/ MEK/ERK pathway has different effects on growth, the prevention of apoptosis, cell cycle arrest, and the induction of drug resistance in cells of various lineages. This may be due to the presence of functional p53 and PTEN and the expression of lineage-specific factors.

\section{Chemotherapy}

Cyclophosphamide. Cyclophosphamide is an alkylating agent and a derivative of nitrogen mustard. A cyclic phosphamidic ester of mecloretamina, it was initially synthesized to improve the selectivity of nitrogenated mustard. It is an odorless, fine white crystalline powder with a molecular weight of 261.1 and a melting point of $49.5-53^{\circ} \mathrm{C}$, and is soluble in water and ethanol, slightly soluble in benzene, ethylene glycol, carbon tetrachloride and dioxane, and sparingly soluble in diethyl ether and acetone. Its log octanol-water partition coefficient is 0.63. Cyclophosphamide reacts with strong oxidizing agents, is sensitive to moisture and light, and is hydrolyzed in aqueous solutions above $30^{\circ} \mathrm{C}$. It is a clinically implemented cytotoxic agent that is effective on a wide range of tumor types, including breast and small-cell lung cancers. Many tumoral cells are, in fact, full of phosphamidasis and phosphatasis, the enzymatic systems responsible for its activation. The hepatic metabolism converts cyclophosphamide to 4-hydroxycyclophosphamide, the tautomerization of which yields aldophosphamide. Acrolein and $\mathrm{N}, \mathrm{N}$-bis-2-(2 chloroethyl) phosphorodiamidate are produced by the spontaneous cleavage of aldophosphamide. $\mathrm{N}, \mathrm{N}$-bis-2-(2 chloroethyl) phosphorodiamidate is a bifunctional alkylating agent and is considered to be the true active agent of cyclophosphamide. DNA is alkylated by $N, N$-bis-2-(2 chloroethyl) phosphorodiamidate at multiple sites. The $N 7$ position of guanine is a site that is particularly susceptible to alkylation by $N, N$-bis-2-(2 chloroethyl) phosphorodiamidate. Alkylation of the $N 7$ position of guanine caused by cyclophosphamide treatment stabilizes the enol tautomer of guanine, which causes guanine to pair with thymine instead of cytosine. The mechanism of action of cyclophosphamide is linked to its interaction with DNA. The most important actions are those which alter the basic mechanisms of growth with mitotic, differentiation and cell functions. The focus on this drug is precisely related to its ability to interfere with the normal mitosis and cell differentiation of all tissues in rapid proliferation.

Cyclophosphamide is used as an adjuvant therapy, often in association with patterns and sequences of combination therapy with fluorouracil and methotrexate (MTX), to treat women who do not have hormone receptors or who are at high risk for relapse. We have identified a gene signature that can predict tumor response to cyclophosphamide and warrants clinical validation (45).

Methotrexate. Antimetabolites prevent cell division because they are embedded in the material of nuclear neosynthesis, or because they combine it irreversibly with the enzymes necessary for life, preventing normal cellular mitotic division. MTX belongs to the class of antimetabolites and folic acid analogs. DNA synthesis requires thymidine 5'-triphosphate (TTP), which is synthesized from thymidine 5'-monophosphate (TMP). Thymidylate synthetase generates TMP by catalyzing the transfer of a methyl group from N5,N10-methylenetetrahydrofolate to 2'-deoxyuridine 5'- monophosphate (dUMP). Treatment with MTX blocks TMP synthesis by preventing the synthesis of N5,N10-methylenetetrahydrofolate. MTX contains a single glutamic acid residue. Folylpoly- $\gamma$-glutamate synthetase (FPGS) catalyzes the addition of one or more glutamic acid moieties to MTX. MTX and its polyglutamylated derivatives inhibit dihydrofolate (DHF) reductase (DHFR). DHFR reduces folate to DHF, which is in turn reduced to tetrahydrofolate. Serine hydroxy-methyltransferase converts tetrahydrofolate to N5,N10-methylenetetrahydrofolate. MTX 
treatment reduces TMP production by eliminating a source of N5,N10-methylenetetrahydrofolate synthesis (13).

5-Fluorouracil. 5-Fluorouracil (5-FU) is also an antimetabolite that is part of similar pyrimidine, capable of preventing the biosynthesis of nucleotide pyrimidines. The 5-FU molecule, inactive in both normal and tumor cells, acquires its cytotoxic activity following biotransformation affected within the cell. Treatment with 5-FU prevents the synthesis of TMP. Thymidylate synthetase is irreversibly inhibited by 5-fluoro-2'deoxyuridine 5'-monophosphate (FdUMP), which is produced by 5-FU. Reduction in TMP levels by treatment with methotrexate or 5-FU inhibits TTP production. This blocks cell proliferation by preventing DNA synthesis.

Anthracyclines. Anthracyclines are anticancer agents belonging to the category of cytotoxic antibiotics. They are a group of drugs, for the most part isolated from natural sources, whose antineoplastic action is due to interaction with DNA. The resulting introduction of nucleic damage triggers apoptosis. Anthracyclines are very effective but also very toxic, unable to discriminate between malignant and healthy cells. They are intercalant agents: aglycone intercalate between DNA base pairs, deforming the double helix. Drug intercalation is necessary but not sufficient for topoisomerase II poisoning. Indeed, the operation of anthracyclines is essential to the formation of a stable complex of DNA-topoisomerase II, which determines the inhibition of this enzyme. Several anthracyclines interfere with topoisomerase II functions by stabilizing an intermediate reaction, in which DNA strands are cut and covalently linked to the tyrosine residues of the enzyme (46). Disruption of the DNA structure by anthracyclines inhibits the synthesis of both DNA and RNA. The antitumor activity of anthracycline drugs results from a combination of various intracellular effects. The main mechanisms of anthracycline-mediated tumor cell death involve i) DNA topoisomerase II inhibition, ii) DNA damage through a p53-dependent and/or p53-independent mechanism, iii) induction of apoptosis mediated by cytochrome c release, iv) proteasome interactions, and v) oxidative damage mediated by the generation of free radicals (47).

DNA cleavage likely makes an important contribution to the cytotoxicity of anthracyclines, as reduced expression of topoisomerase II $\alpha$ is correlated with decreased anthracycline sensitivity (48). These anthracyclines induce apoptotic cell death. In breast cancer, the anthracyclines used are doxorubicin (Adriamycina) and epirubicin.

Taxanes. Microtubule-targeting agents have made significant contributions to cancer therapy over the past fifty years. Taxanes have been used to treat a broad range of malignancies, including leukemias and lymphomas, and many types of solid tumors. They have frequently been used in the treatment of advanced ovarian, breast, lung, head and neck and prostate cancer, and are increasingly being used in early-stage disease (49).

Taxanes comprise a group of drugs which includes paclitaxel (Taxol) and docetaxel (Taxotere), used in the treatment of cancer. They have a unique way of preventing the growth of cancer cells, by affecting cell structures called microtubules that play an important role in cell functions. In normal cell growth, microtubules are formed when a cell starts dividing. Once the cell stops dividing, these microtubules are broken down or destroyed. Taxanes, however, stop the microtubules from breaking down. Thus, cancer cells become so clogged with microtubules that they cannot grow and divide (50).

Epothilones are a novel class of antineoplastic agents possessing antitubulin activity. They induce tubulin polymerization and enhance microtubule stability. Epothilone-induced stabilisation of microtubules has been shown to cause arrest at the $\mathrm{G} 2 / \mathrm{M}$ transition of the cell cycle, as well as apoptosis. The compounds are active against cancer cells that have developed resistance to taxanes resulting from the acquisition of $\beta$-tubulin overexpression or mutations, and are active against multidrugresistant cells that overexpress P-glycoprotein or multidrug resistance-associated protein. Thus, epothilones represent a new class of antimicrotubule agents with low susceptibility to key tumor resistance mechanisms (51).

\section{Hormone therapy}

Aromatase inhibitors. Many breast cancers rely on supplies of the hormone oestrogen to grow. In women who have been through menopause, oestrogen is mainly acquired through the changing of androgens (sex hormones produced by the adrenal glands) into oestrogen. This is carried out by an enzyme called aromatase, with the conversion process, known as aromatisation, taking place mainly in the fatty tissues of the body. Aromatase cytochrome P450 (CYP19) converts androgen to estrogen (52). Enzyme aromatase, which catalyzes the reaction of estrogen synthesis starting from androgens, in particular by the conversion of androstenedione estrone and estradiol to testosterone, is inhibited by aromatase inhibitors. Aromatase inhibitors prevent the proliferation of breast cancer cells by blocking estrogen production. There are two classes of aromatase inhibitors, which differ in chemical structure and their mechanism of action. Type 1 inhibitors (enzyme inhibitors and steroids), such as exemestane, bind irreversibly to aromatase. Type 2 inhibitors (non-steroidal enzyme inhibitors), such as anastrozole and letrozole, reversibly bind to aromatase. In one comparative study conducted in 2000 , anastrozole satisfied the predefined criteria for equivalence to tamoxifen (TAM). Moreoever, both a significant increase in thymidine 5'-triphosphate (TTP) and a lower incidence of thromboembolic events and vaginal bleeding with anastrozole was observed (53). Aromatase inhibitors provide an alternative to TAM as an adjuvant therapy for post-menopausal, hormonereceptor-positive breast cancer patients (54).

Tamoxifen. Hormonal intervention as a means to treat breast cancer has been implemented since the late 1800's, when Beatson (55) was able to treat this cancer by ovariectomy. By 1900, Boyd (56) had reported that $37 \%$ of breast cancer patients studied responded to ovariectomy. Many years later, the mechanisms underlying this response were demonstrated to be related to the fact that a large subset of breast cancers are hormonally responsive due to their dependence on signaling via the estrogen receptor (ER) (57). TAM, a non-steroidal antiestrogen, is currently used to treat post-menopausal breast cancer patients. In estrogen-sensitive breast cancer cell lines, TAM and some of its metabolites display, for the induction 
of estrogen-regulated proteins, a partial agonist/antagonist activity. They also totally inhibit the proliferation of these cells (58). The observed clinical efficacy of TAM has been attributed to both growth arrest and the induction of apoptosis within breast cancer cells. Although its primary mechanism of action is believed to be related to the inhibition of estrogen receptor (ER), research over the years has indicated that additional, non-ER-mediated mechanisms exist. These include the modulation of signaling proteins, such as protein kinase $C$ (PKC), calmodulin, transforming growth factor- $\beta$ (TGF-B), and the proto-oncogene c-myc. Recent studies have implicated the role of caspases and MAPK, including c-Jun Nterminal kinase and p38, in TAM-induced apoptotic signaling. Oxidative stress, mitochondrial permeability transition, ceramide generation, as well as changes in cell membrane fluidity may also play important roles in TAM-induced apoptosis (59). TAM has significantly decreased the mortality rate of both premenopausal and postmenopausal women with hormone receptor-positive breast cancer (60).

\section{Antibody-based therapy}

The term 'targeted therapy' refers to a new generation of cancer drugs designed to interfere with a specific molecular target, typically a protein, believed to play a critical role in tumor growth or progression. Over the last several years, multiple definitions for the term targeted therapy have emerged. First, the US Food and Drug Administration (FDA) considers targeted therapy to be a drug with an approved label with a specific reference to a simultaneously- or previouslyapproved diagnostic test that must be performed before the patient can be considered eligible to receive the drug (61). For many scientists and oncologists, targeted therapy is defined as a drug with a focused mechanism that acts specifically on a well-defined target or biologic pathway which, when inactivated, causes the regression or destruction of the malignant process. Many investigators consider anticancer antibodies an additional type of targeted therapy, especially when they are conjugated with cytotoxic radioisotopes, cytotoxic drugs or cellular poisons that seek out and kill malignant cells bearing the target antigen (62).

Genetic polymorphisms in drug-metabolizing enzymes, transporters, receptors and other drug targets have been linked to inter-individual differences in response to the efficacy and toxicity of many medications. Pharmacogenomic studies are rapidly elucidating the inherited nature of these differences in drug disposition and effect, thereby enhancing drug discovery and providing a stronger scientific basis for optimizing drug therapy on the basis of the genetic constitution of each patient (63).

Trastuzumab. Trastuzumab is the first example of a novel approach to genetic and biologic research concerning neoplastic cell growth; it is a rare example of a success story of clinical and translational medicine moving from bedside to bench to bedside (64). Trastuzumab (Herceptin ${ }^{\circledR}$, Genentech Inc., San Francisco, CA) is a recombinant humanized monoclonal antibody directed against the extracellular domain of the human epidermal growth factor receptor 2 (HER2 or ErbB-2) protein. Currently, trastuzumab is the only HER2-targeted therapy approved by the FDA for the treatment of metastatic breast cancer (65). Signal transduction research has shown the importance of members of the human epidermal growth factor receptor (HER) family of transmembrane tyrosine kinases in a number of solid tumors. One member of this family is HER2 (ErbB-2). HER2 is normally expressed in all epithelial cells but, in a percentage of breast cancer cases ranging from 10 to $30 \%$, was amplified, with a consequent membrane glycoprotein overexpression. In a small percentage of cases, ranging from 3 to $10 \%$, hyper-expression of glycoprotein without gene amplification was observed. The mechanism behind this is still unknown. The gene for this receptor is amplified in up to $30 \%$ of breast cancers, leading to aggressive behavior and an unfavorable prognosis. Amplification of the HER-2/neu gene is a significant predictor of both overall survival and time to relapse in patients with breast cancer (66). However, the overexpressed HER2 receptor protein also serves as a target for anti-HER2 antibody (trastuzumab) therapy. The presence or absence of amplification can be used to differentiate patients who may have a response to the antibody from those who will not have a response (67). Unlike most pathologic testing, which serves as an adjunct to establishing a diagnosis, the results of HER2 testing stand alone in determining which patients are likely to respond to trastuzumab, a monoclonal antibody against HER2. Given the significant clinical impact of testing results on subsequent patient management, the accuracy, precision and reproducibility of HER 2 testing are critical. At present, several pre-analytic factors, including the time from tissue removal to tissue fixation, are underappreciated as important variables that have the potential to negatively impact the consistency and reliability of HER2 testing. Rigorous quality control and standardization of the testing process, from the handling of tissue samples to the interpretation and reporting of results, are essential for achieving accurate and reproducible assay results (68).

The principal adverse event associated with trastuzumab therapy among patients with prior exposure to anthracycline is cardiac dysfunction (CD). Patients treated with trastuzumab appear to be at an increased risk of developing of cardiomyopathy, reminiscent of the cardiomyopathy associated with anthracyclines. The pathophysiologic basis of trastuzumabassociated CD is poorly understood. It remains unknown whether trastuzumab exacerbates previous damage caused by anthracyclines or acts through an independent mechanism to directly affect the cardiac myocyte. It has been suggested that p185HER2 may play a role in myocyte survival. However, the molecular mechanisms underlying trastuzumab-associated cardiomyopathy are obscure. The greatest risk for the development of CD was observed in patients receiving concomitant trastuzumab and anthracycline. Risks for patients treated with concurrent paclitaxel and trastuzumab and patients treated with trastuzumab as a single agent seem to be substantially lower than those associated with the concurrent administration of anthracycline and trastuzumab (69). Trastuzumab is generally well tolerated by most patients, the most significant adverse effects being acute fever and/or chills and the potential to cause CD. Serious adverse events, including anaphylaxis and death, have occurred in $0.25 \%$ of patients. Symptomatic or asymptomatic CD has occurred in $27 \%$ of patients receiving anthracycline and cyclophosphamide treatment combined with 
trastuzumab. Thus, combination therapy with anthracyclines is not recommended. Symptomatic or asymptomatic CD occurred in $13 \%$ of patients receiving trastuzumab plus paclitaxel and in $4.7 \%$ of patients receiving trastuzumab alone (70).

\section{Mechanisms of resistance and the RAF/MEK/ERK pathway}

Inter-individual variability in drug response and the emergence of adverse drug reactions are the main causes of treatment failure in cancer therapy. Recently, membrane transporters have been recognized as important determinants of drug disposition, thereby affecting chemosensitivity and resistance. Genetic factors contribute to inter-individual variability in drug transport and targeting. Pharmacogenetic studies of membrane transporters can therefore lead to new approaches to optimizing cancer therapy (71). MAP-kinase activity and expression may be increased in some human breast cancers, independently of EGFR or HER2, rendering these tumors less sensitive to anticancer approaches targeted to HER receptors (72). A potential mechanism by which resistance to targeted antibodies might develop is via the disruption of the therapeutic agent in binding to the target protein (65). The membrane-associated glycoprotein mucin-4 might mask HER2, preventing it from binding effectively to trastuzumab (73). An important study provided compelling evidence supporting a role for the PI3K/Akt pathway in trastuzumab resistance. The authors essentially showed that, in patients with HER2-overexpressing breast tumors, the absence of PTEN expression was associated with a poorer response to trastuzumab-based therapy than in those patients with normal PTEN. Furthermore, it has been demonstrated that, in PTENdeficient cells, PI3K inhibitors rescue trastuzumab resistance in vitro and in vivo (74). These results suggest that PTEN loss could serve as a predictor of trastuzumab resistance, and that PI3K inhibitors should be explored as potential therapies in patients with trastuzumab-resistant tumors expressing low levels of PTEN protein (65).

A study performed to investigate which pathway plays a major role in Ras-induced drug resistance to chemotherapeutic agents in breast cancer cells demonstrated that, although inhibition of MEK/MAPK or PI3K/Akt can enhance the cytotoxicity of paclitaxel, doxorubicin or 5-FU, inhibition of the PI3K/Akt pathway seems to have a greater effect than inhibition of the MEK/MAPK pathway in reversing Rasmediated drug resistance. The results indicate that the PI3K pathway may play a more important role in receptor tyrosine kinase-mediated resistance to chemotherapy, and suggest that PI3K/Akt may be a critical target molecule for anticancer intervention in breast cancer (75). Therefore, MAP-kinase may be an additional target that anti-EGFR and anti-HER2 interventions may have to disable in order to exert an antitumor effect (76).

\section{Conclusions}

The biologic heterogeneity of breast cancer has been elucidated through genome-wide profiling technologies. Several studies have defined the genetic inter-individual differences among breast cancer patients. These differences may affect response to therapy and prognosis. Mechanisms of resistance to therapy may be identified by exploring alterations of genes involved in breast cancer development. Therefore, a better understanding of breast cancer biology may enable oncologists to identify a tailored treatment for these patients.

\section{Acknowledgments}

This study was supported in part by Ricerca Sanitaria 2007 Assessorato della Sanità, Regione Sicilia.

\section{References}

1. Goetz MP, Kamal A and Ames MM: Tamoxifen pharmacogenomics: the role of CYP2D6 as a predictor of drug response. Clin Pharmacol Ther 83: 160-166, 2008.

2. Navolanic PM, Steelman LS and McCubrey JA: EGFR family signaling and its association with breast cancer development and resistance to chemotherapy (Review). Int J Oncol 22: 237-252, 2003.

3. Bonadonna G, Robustelli Della Cuna G and Valagussa P (eds): Medicina Oncologica. 8th edition, Elsevier Masson, Milano, 2007.

4. Guarneri V and Conte PF: The curability of breast cancer and the treatment of advanced disease. Eur J Nucl Med Mol Imaging 31: S149-S161, 2004.

5. Hussain SA, Palmer DH, Spooner D and Rea DW: Molecularly targeted therapeutics for breast cancer. BioDrugs 21: 215-224, 2007.

6. Chabner BA: Biological basis for cancer treatment. Ann Intern Med 118: 633-637, 1993.

7. Ford D, Easton, DF, Stratton M, et al: Genetic heterogeneity and penetrance analysis of the BRCA1 and BRCA2 genes in breast cancer families. Am J Hum Genet 62: 676-689, 1998.

8. Hall JM, Lee MK, Newman B, Morrow JE, Anderson LA, Huey B and King MC: Linkage of early-onset familial breast cancer to chromosome 17q21. Science 250: 1684-1689, 1990.

9. Miki Y, Swensen J, Schattuck-Eidens D, et al: A strong candidate for the breast and ovarian cancer susceptibility gene BRCA1. Science 266: 66-71, 1994.

10. Wooster R, Neuhausen S, Manigion J, et al: Localisation of a breast cancer susceptibility gene (BRCA2) to chromosome 13q12-13. Science 265: 2088-2090, 1994.

11. Wooster R, Bignell G, Lancaster J, et al: Identification of the breast cancer susceptibility gene BRCA2. Nature 378: 789-792, 1995.

12. Tavtigian SV, Simard J, Rommens J, et al: The complete BRCA2 gene and mutations in chromosome 13q-linked kindreds. Nat Genet 12: 333-337, 1996.

13. Steelman LS, Navolanic PM, Franklin RA, Bonati A, Libra M, Stivala F, Martelli AM and McCubrey JA: Combining chemo-, hormonal and targeted therapies to treat breast cancer (Review). Mol Med Rep 1: 139-160, 2008.

14. Centers for Disease Control and Prevention: Breast cancer: symptoms. http://www.cdc.gov/cancer/breast/basic_info/symptoms.htm, 2006.

15. Tay YC, Domchek S, Parmigiani G and Chen S: Breast cancer risk among male BRCA1 and BRCA2 mutation carriers. J Natl Cancer Inst 99: 1811-1814, 2007.

16. Lane D: Anthony Dipple Carcinogenesis Award. p53 from pathway to therapy. Carcinogenesis 25: 1077-1081, 2004.

17. Cheah PL and Looi LM: p53: an overview of over two decades of study. Malays J Pathol 23: 9-16, 2001.

18. Monti P, Ciribilli Y, Jordan J, Manichini P, Umbach DM, Resnick MA, Luzzatto L, Inga A and Fronza G: Transcriptional functionality of germ line p53 mutants influences cancer phenotype. Clin Cancer Res 13: 3789-3795, 2007.

19. Lynch HT, Silva E, Snyder C and Lynch JF: Hereditary breast cancer: part I. Diagnosing hereditary breast cancer syndromes. Breast J 14: 3-13, 2008.

20. Li X, Lin G, Wu B, Zhou X and Zhou K: Overexpression of PTEN induces cell growth arrest and apoptosis in human breast cancer ZR-75-1 cells. Acta Biochim Biophys Sin (Shanghai) 39: 745-750, 2007.

21. Teresi RE, Zbuk KM, Pezzolesi MG, Waite KA and Eng C: Cowden syndrome-affected patients with PTEN promoter mutations demonstrate abnormal protein translation. Am J Hum Genet 81: 756-767, 2007. 
22. Haiman CA, Stram DO, Cheng I, Giorgi EE, Pooler L, Penney K, Le Marchand L, Henderson BE and Freedman ML: Common genetic variation at PTEN and risk of sporadic breast and prostate cancer. Cancer Epidemiol Biomarkers Prev 15: 1021-1025, 2006.

23. Gustafson S, Zbuck KM, Scacheri C and Eng C: Cowden syndrome. Semin Oncol 34: 428-434, 2007.

24. Shen WH, Balajee AS, Wang J, Wu H, Eng C, Pandolfi PP and Yin Y: Essential role for nuclear PTEN in maintaining chromosomal integrity. Cell 128: 157-170, 2007.

25. Pfeiler G, Horn F, Lattrich C, Klappenberger S, Ortmann O and Treeck O: Apoptotic effects of signal transduction inhibitors on human tumor cells with different PTEN expression. Oncol Rep 18: 1305-1309, 2007

26. Smith TR, Miller MS, Lohman K, Lange EM, Case LD, Mohrenweiser HW and Hu JJ: Polymorphisms of XRCC1 and XRCC3 genes and susceptibility to breast cancer. Cancer Lett 190: 183-190, 2003.

27. Terry MB, Gammon MD, Zhang FF, Knight JA, Wang Q, Britton JA, Teitelbaum SL, Neugut AI and Santella RM: ADH3 genotype, alcohol intake and breast cancer risk. Carcinogenesis 27: 840-847, 2006.

28. Zhang J and Liu Y: HER2 over-expression and response to different chemotherapy regimens in breast cancer. J Zhejiang Univ Sci B: 5-9, 2008.

29. Révillion F, Lhotellier V, Hornez L, Bonneterre J and Peyrat JP: ErbB/HER ligands in human breast cancer, and relationships with their receptors, the bio-pathological features and prognosis. Ann Oncol 19: 73-80, 2008.

30. Shelton JG, Steelman LS, Abrams SL, Bertrand FE, Franklin RA, McMahon M and McCubrey JA: The epidermal growth factor receptor gene family as a target for therapeutic intervention in numerous cancers: what's genetics got to do with it? Expert Opin Ther Targets 9: 1009-1030, 2005.

31. Vanden Bempt I, Drijkoningen M and De Wolf-Peeters C: The complexity of genotypic alterations underlying HER2-positive breast cancer: an explanation for its clinical heterogeneity. Curr Opin Oncol 19: 552-557, 2007.

32. Pal SK and Pegram M: HER2 targeted therapy in breast cancer... beyond Herceptin. Rev Endocr Metab Disord 8: 269-277, 2007.

33. McCubrey JA, Steelman LS, Chappel WH: Roles of the RAF/ MEK/ERK pathway in cell growth, malignant transformation and drug resistance. Biochim Biophys Acta 1773: 1263-1284, 2006.

34. Garnett MJ and Marais R: Guilty as charged: B-RAF is a human oncogene. Cancer Cell 6: 313-319, 2004.

35. Nakamura M, Han B, Nishishita T, Bai Y and Kakudo K: Calcitonin targets extracellular signal-regulated kinase signaling pathway in human cancers. J Mol Endocrinol 39: 375-384, 2007.

36. Zheng L, Ren JQ, Zhang L, Chen Q and Zhu HG: Over-expression of HER 2/neu downregulates wild p53 protein expression via PI3K and Ras/Raf/MEK/ERK pathways in human breast cancer cells. Zhonghua Bing Li Xue Za Zhi 33: 358-362, 2004.

37. McCubrey JA, Steelman LS, Abrams SL, et al: Roles of the RAF/MEK/ERK and PI3K/PTEN/AKT pathways in malignant transformation and drug resistance. Adv Enzyme Regul 46: 249-279, 2006.

38. Chang F, Steelman LS, Shelton JG, et al: Regulation of cell cycle progression and apoptosis by the Ras/Raf/MEK/ERK pathway (Review). Int J Oncol 22: 469-480, 2003.

39. Chang F, Lee JT, Navolanic PM, Steelman LS, Shelton JG, Blalock WL, Franklin RA and McCubrey JA: Involvement of PI3K/Akt pathway in cell cycle progression, apoptosis, and neoplastic transformation: a target for cancer chemotherapy. Leukemia 17: 590-603, 2003.

40. Lai YL, Mau BL, Cheng WH, Chen HM, Chiu HH and Tzen CY: PIK3CA exon 20 mutation is independently associated with a poor prognosis in breast cancer patients. Ann Surg Oncol 15: 1064-1069, 2008

41. Adami HO, Malker B, Holmberg L, Persson I and Stone B: The relation between survival and age at diagnosis in breast cancer. N Engl J Med 315: 559-563, 1986.

42. Nixon AJ, Neuberg D, Hayes DF, et al: Relationship of patient age to pathologic features of the tumor and prognosis for patients with stage I or II breast cancer. J Clin Oncol 12: 888-894, 1994.

43. Anders CK, Acharya CR, Hsu DS, et al: Age-specific differences in oncogenic pathway deregulation seen in human breast tumors. PLOS ONE 3: e1373, 2008.
44. Wang JM, Chao JR, Chen W, Kuo ML, Yen JJ and Yang-Yen HF: The antiapoptotic gene mcl-1 is up-regulated by the phosphatidylinositol 3-kinase/Akt signaling pathway through a transcription factor complex containing CREB. Mol Cell Biol 19: 6195-6206, 1999.

45. Korrat A, Greiner T, Maurer M, Metz M and Fiebig HH: Gene signature-based prediction of tumor response to cyclophosphamide. Cancer Genomics Proteomics 4: 187-195, 2007.

46. Binaschi M, Bigioni M, Cipollone A, Rossi C, Goso C, Maggi CA, Capranico G and Animati F: Anthracyclines: selected new developments. Curr Med Chem Anticancer Agents 1: 113-130, 2001.

47. Choi JY, Nowell SA, Blanco JG and Ambrosone CB: The role of genetic variability in drug metabolism pathways in breast cancer prognosis. Pharmacogenomics 7: 613-624, 2006.

48. Järvinen TA, Tanner M, Rantanen V, Bärlund M, Borg A, Grénman S and Isola J: Amplification and deletion of topoisomerase IIalpha associate with ErbB-2 amplification and affect sensitivity to topoisomerase II inhibitor doxorubicin in breast cancer. Am J Pathol 156: 839-847, 2000.

49. Zelnak AB: Clinical pharmacology and use of microtubuletargeting agents in cancer therapy. Methods Mol Med 137: 209-234, 2007.

50. McGrogan BT, Gilmartin B, Carney DN and McCann A: Taxanes, microtubules and chemoresistant breast cancer. Biochim Biophys Acta 1785: 96-132, 2008.

51. Reichenbach H and Höfle G: Discovery and development of the epothilones: a novel class of antineoplastic drugs. Drugs R D 9: $1-10,2008$.

52. Hong Y, Cho M, Yuan YC and Chen S: Molecular basis for the interaction of four different classes of substrates and inhibitors with human aromatase. Biochem Pharmacol 75: 1161-1169, 2008.

53. Nabholtz JM, Buzdar A, Pollak M, Harwin W, Burton G, Mangalik A, Steinberg M, Webster A and von Euler M: Anastrozole is superior to tamoxifen as first-line therapy for advanced breast cancer in postmenopausal women: results of a North American multicenter randomized trial. Arimidex Study Group. J Clin Oncol 18: 3758-3767, 2000.

54. Eisen A, Trudeau M, Shelley W, Messersmith H and Pritchard KI: Aromatase inhibitors in adjuvant therapy for hormone receptor positive breast cancer: A systematic review. Cancer Treat Rev 34: 157-174, 2008.

55. Beatson GT: On the treatment of inoperable cases of carcinoma of the mammary: suggestions for a new method of treatment with illustrative cases. Lancet 2: 104-107, 162-165, 1896.

56. Boyd S: On oophorectomy in cancer of the breast. BMJ 2: 1161-1167, 1900 .

57. Jensen EV and Jacobson HI: Basic guides to the mechanism of estrogen action. Recent Prog Horm Res 18: 387-414, 1962.

58. Borgna JL and Rochefort $\mathrm{H}$ : Effects and mechanism of action of antiestrogens in breast cancer. Sem Hop 60: 703-709, 1984.

59. Mandlekar S and Kong AN: Mechanisms of tamoxifen-induced apoptosis. Apoptosis 6: 469-477, 2001.

60. Iino Y and Koibuchi Y: Clinical benefit of antiestrogens for breast cancer. Nippon Rinsho 66: 168-173, 2008.

61. US Food and Drug Administration, Office of Combination Products: Federal Food, Drug, and Cosmetic Act, as amended by the Medical Device User Fee and Modernization Act of 2002. National Press Office, Rockville, MD, 2003.

62. Ross JS, Schenkein DP, Pietrusko R, et al: Targeted therapies for cancer 2004. Am J Clin Pathol 122: 598-609, 2004.

63. Ewans WE and Relling MV: Pharmacogenomics: translating functional genomics into rational therapeutics. Science 286: 487-491, 1999.

64. Munshi A, Singh P and Jalali R: Trastuzumab: is the new evidence revolutionary? J Cancer Res Ther 2:144-146, 2006.

65. Nahta R, Yu D, Hung MC, Hortobagyi GN and Esteva FJ: Mechanisms of disease: understanding resistance to HER2-targeted therapy in human breast cancer. Nat Clin Pract Oncol 3: 269-280, 2006.

66. Slamon DJ, Clark GM, Wong SG, Levin WJ, Ullrich A and McGuire WL: Human breast cancer: correlation of relapse and survival with amplification of the HER-2/neu oncogene. Science 235: 177-182, 1987

67. Green MR: Targeting targeted therapy. N Eng J Med 350: 2191-2193, 2004 .

68. Hicks DG and Kulkarni S: HER2+ breast cancer: review of biologic relevance and optimal use of diagnostic tools. Am J Clin Pathol 129: 263-273, 2008. 
69. Seidman A, Hudis C, Pierri MK, Shak S, Paton V, Ashby M, Murphy M, Stewart SJ and Keefe D: Cardiac dysfunction in the trastuzumab clinical trial experience. Am J Clin Oncol 20: 1215-1221, 2002.

70. McKeage K and Perry CM: Trastuzumab: a review of its use in the treatment of metastatic breast cancer overexpressing HER2. Drugs 62: 209-243, 2002.

71. Huang Y: Pharmacogenetics/genomics of membrane transporters in cancer chemotherapy. Cancer Metastasis Rev 26: 183-201, 2007.

72. Lenferink AE, Simpson JF, Shawver LK, Coffey RJ, Forbes JT and Arteaga CL: Blockade of the epidermal growth factor receptor tyrosine kinase suppresses tumorigenesis in MMTV/Neu ${ }^{+}$ MMTV/TGF-alpha bigenic mice. Proc Natl Acad Sci USA 97: 9609-9614, 2000.
73. Nagy P, Friedländer E, Tanner M, Kapanen AI, Carraway KL, Isola $\mathrm{J}$ and Jovin TM: Decreased accessibility and lack of activation of ErbB2 in JIMT-1, a herceptin-resistant, MUC4-expressing breast cancer cell line. Cancer Res 65: 473-482, 2005.

74. Nagata $Y$, Lan KH, Zhou X, et al: PTEN activation contributes to tumor inhibition by trastuzumab, and loss of PTEN predicts trastuzumab resistance in patients. Cancer Cell 6: 117-127, 2004. 75. Jin W, Wu L, Liang K, Liu B, Lu Y and Fan Z: Roles of the PI-3K and MEK pathways in Ras-mediated chemoresistance in breast cancer cells. Br J Cancer 89: 185-191, 2003.

76. Cardoso F, Piccart MJ, Durbecq V and Di Leo A: Resistance to trastuzumab: a necessary evil or a temporary challenge? Clin Breast Cancer 3: 247-257, 2002. 